\title{
Pabellón Polideportivo de la Universidad de Liverpool Inglaterra
}

DENYS LASDUN \& PARTNERS, arquitectos

\section{simopsis}

158-3

Se pueden apreciar claramente dos zonas principales: «piscina» $\mathrm{y}$ "pista para deportes». El conjunto cuenta además con cuatro campos de pelota; las salas de actividades generales, que permiten practicar el manejo de pesas, esgrima, gimnasia rítmica, judo, lucha, y otras formas de ejercicio físico; oficinas, vestuarios para ambos sexos, bar, almace-

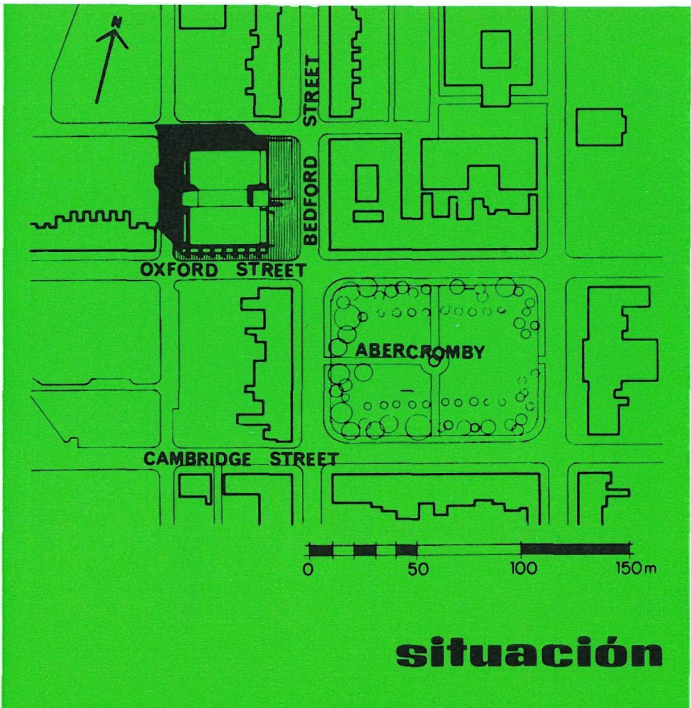
nes, etc.

La piscina mide $33,53 \mathrm{~m} \times 14,63 \mathrm{~m}$; su profundidad oscila entre $0,91 \mathrm{~m}$ y $3,80 \mathrm{~m}$, y dispone de palancas y trampolines y una galería con asientos para 150 espectadores, desde la que se domina el desarrollo de las competiciones deportivas.

En la pista polideportiva, da $36,58 \mathrm{~m} \times 19,50 \mathrm{~m}$, se practican el baloncesto, tenis, etc.; mediante un sistema de redes puede dividirse, con objeto de que se puedan desarrollar en ella, simultáneamente, varias actividades deportivas.

En el cruce de las calles Oxford Street y Bedford Street North, colindante con la Abercromby Square, formando parte del programa de ampliación de las instalaciones universitarias, ha sido construido este Centro Polideportivo, destinado a facilitar la práctica de los deportes, organizar clases de educación física, y proporcionar posibilidades de esparcimiento físico a los estudiantes, a través de sus Clubs universitarios y Asociaciones de estudiantes.

La participación recreativa en este Pabellón puede ser en «forma»: de competiciones interdepartamentales, de clases instructivas sobre la práctica de diferentes deportes, etc. Lo importante es que por su recinto pasan de 3.000 a 4.000 estudiantes por semana, y que en todo momento actúan en él unos 200 estudiantes.

Podemos apreciar claramente en la planificación de este Centro dos zonas principales — «piscina» y "pista para deportes»-, situadas, respectivamente, a uno y otro lado del núcleo central que contiene las zonas deportivas más pequeñas, los vestuarios y las circulaciones verticales, etc.

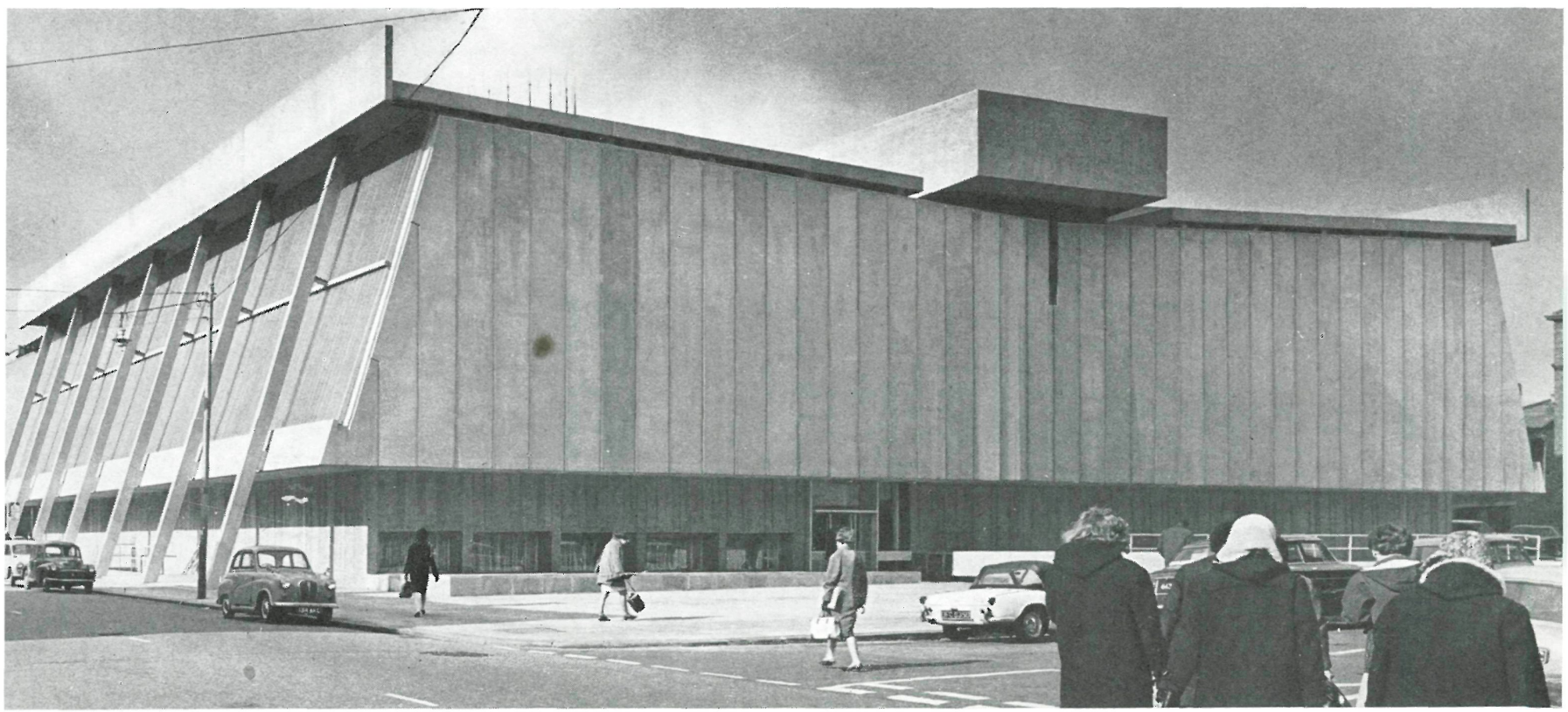




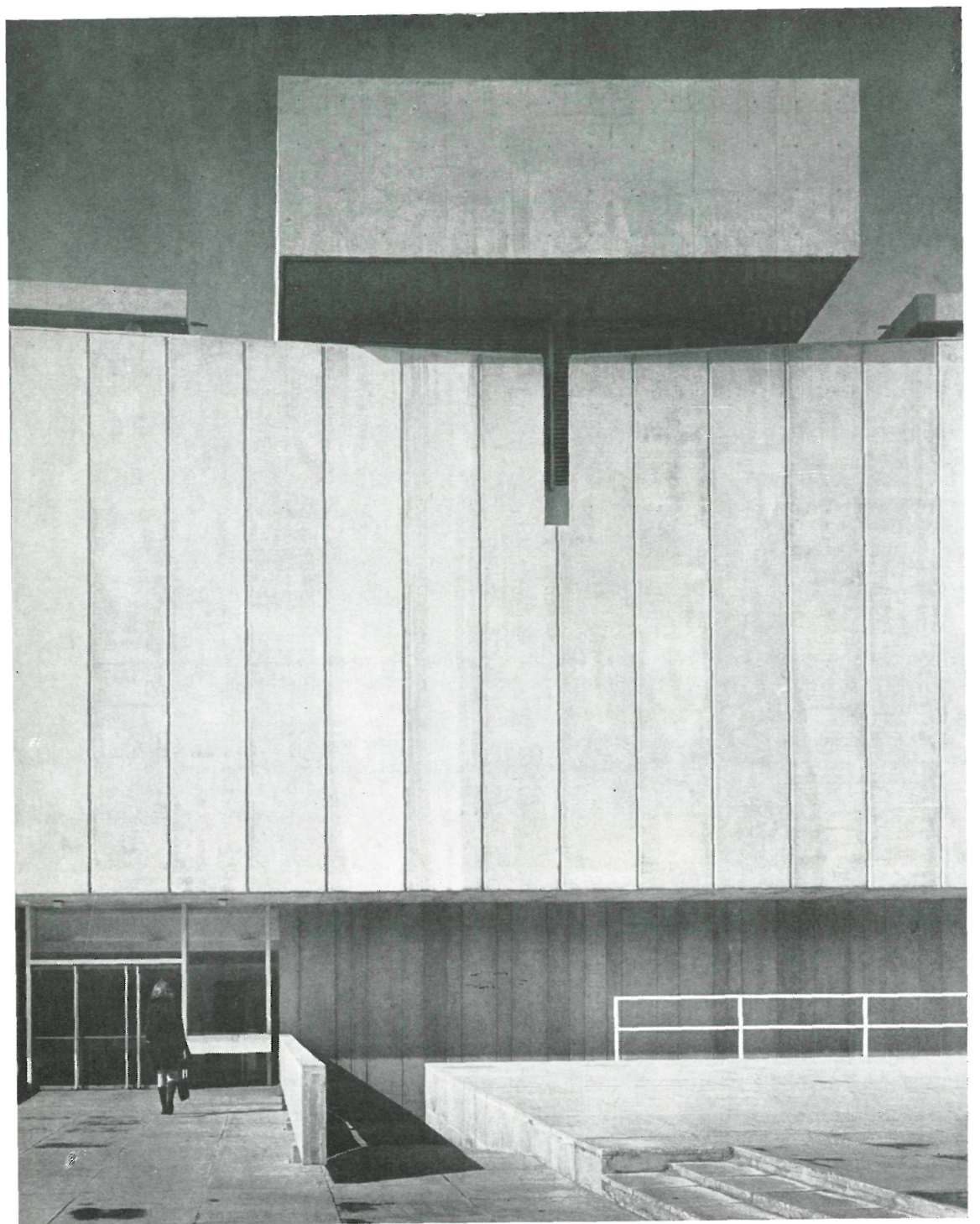

A. Sala de deportes.-B. Piscina.-C. Sala de entrenamiento--D. Estudio.-E. Estancia. F. Pistas de pelota. - G. Restaurante.-H. Galería de la piscina.-I. Muro de escalada. talaciones.

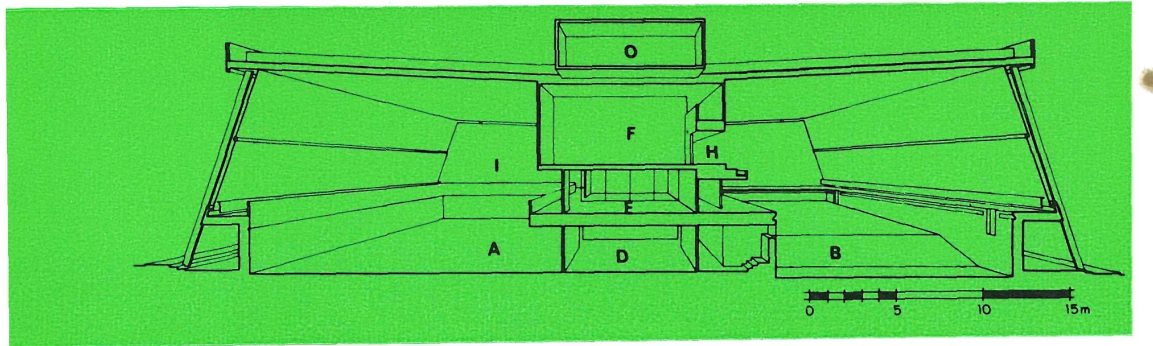

\section{interiomes}

La piscina mide $33,53 \mathrm{~m} \times$ $\times 14,63 \mathrm{~m}$; tiene una profundidad que oscila entre $0,91 \mathrm{~m}$ y $3,80 \mathrm{~m}$, aprovechándose el espacio creado debajo del extremo de menor profundidad de la piscina para situar las oficinas; dispone de 6 calles, y en las paredes han sido previstas dos ventanas para poder observar el movimiento de los nadadores por debajo del agua. Como instalaciones auxiliares de la piscina figuran una palanca de $5 \mathrm{~m}$ de altura, con trampolines a 1 y $2 \mathrm{~m}$ de altura; y una galería, con asientos para 150 espectadores, desde la que se dominan las competiciones deportivas.

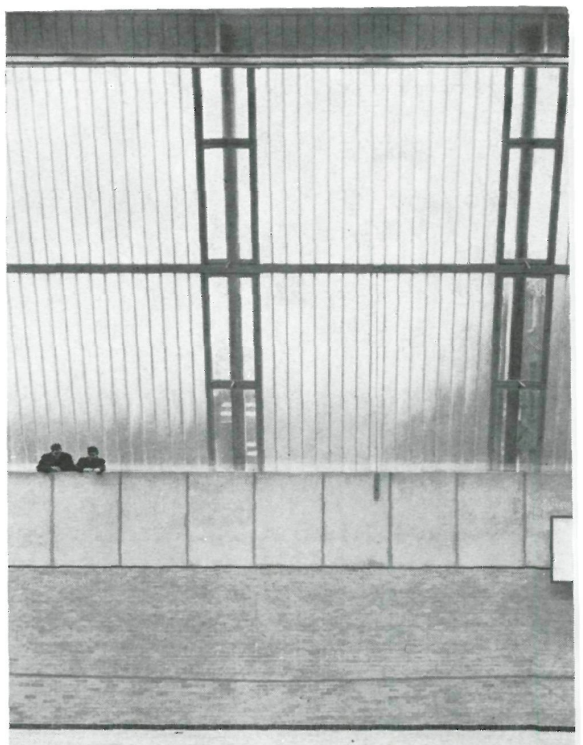

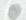




\section{plantas \\ bajo \\ proime eren \\ segumed a}

La pista polideportiva, cuyas dimensiones son $36,58 \mathrm{~m} \times$ $\times 19,50 \mathrm{~m}$, presenta las adecuadas señalizaciones para poder practicar en ella el baloncesto, tenis, etc.; existiendo la posibilidad de subdividirla, mediante redes, con el fin de que se puedan practicar simultáneamente varias actividades cuando sea necesario. En un extremo de la misma están el material y aparatos gimnásticos.

Al nivel de la primera planta de la pista hay una galería desde la cual se puede acceder a la pared de trepa, que es un modelo de relieves de hormigón y ladrillo, proyectada por Donald Mill, y que facilita el aprendizaje de las técnicas de escalada en roca y el manejo de la cuerda.

El conjunto cuenta, además, con cuatro campos de pelota; las salas de actividades generales que permiten practicar el manejo de las pesas, esgrima, distintos estilos de gimnasia rítmica, judo, lucha, y otros deportes; un departamento de oficinas para uso de los miembros del Departamento de Educación Física, vestuarios para ambos sexos, un almacén para equipos, un bar, etc.
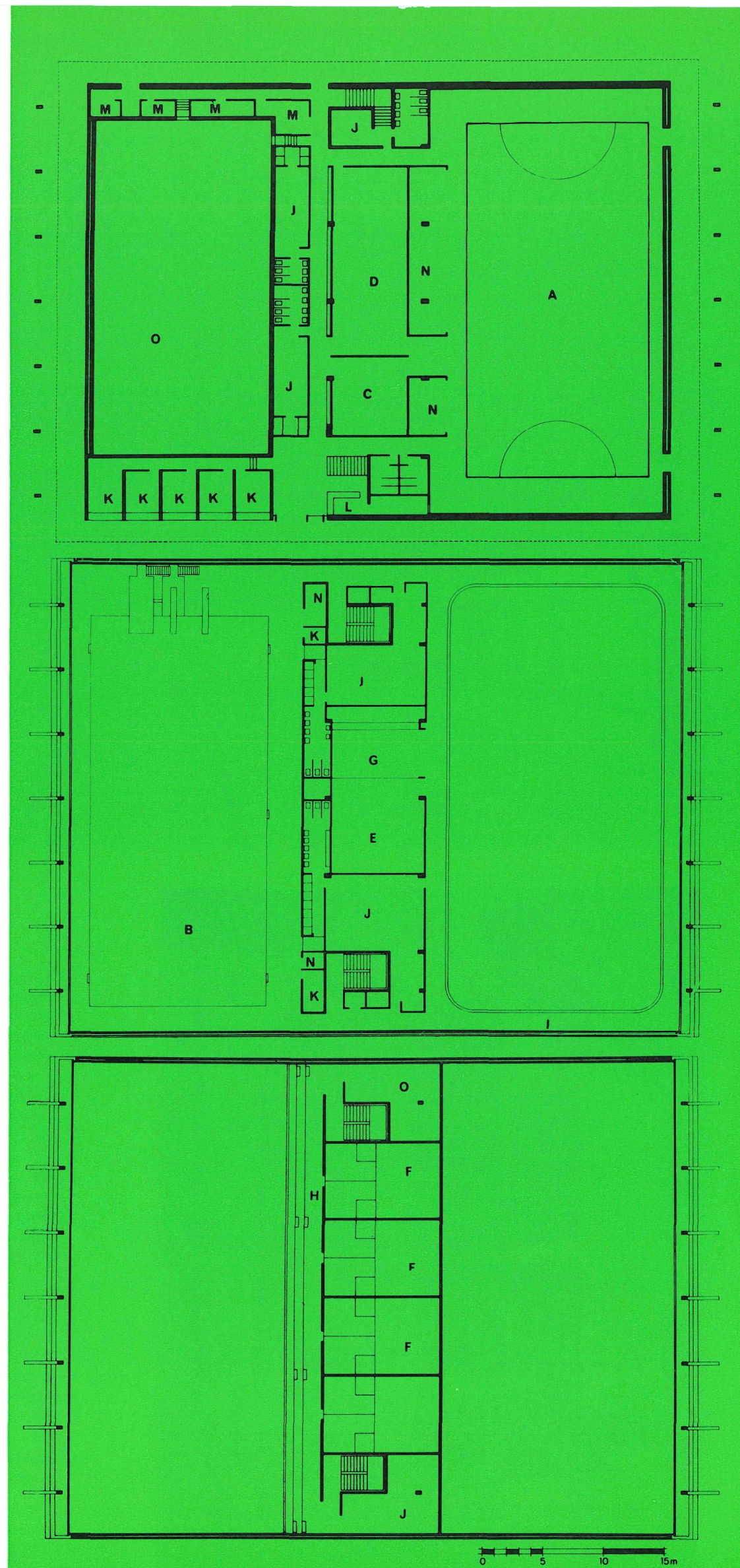

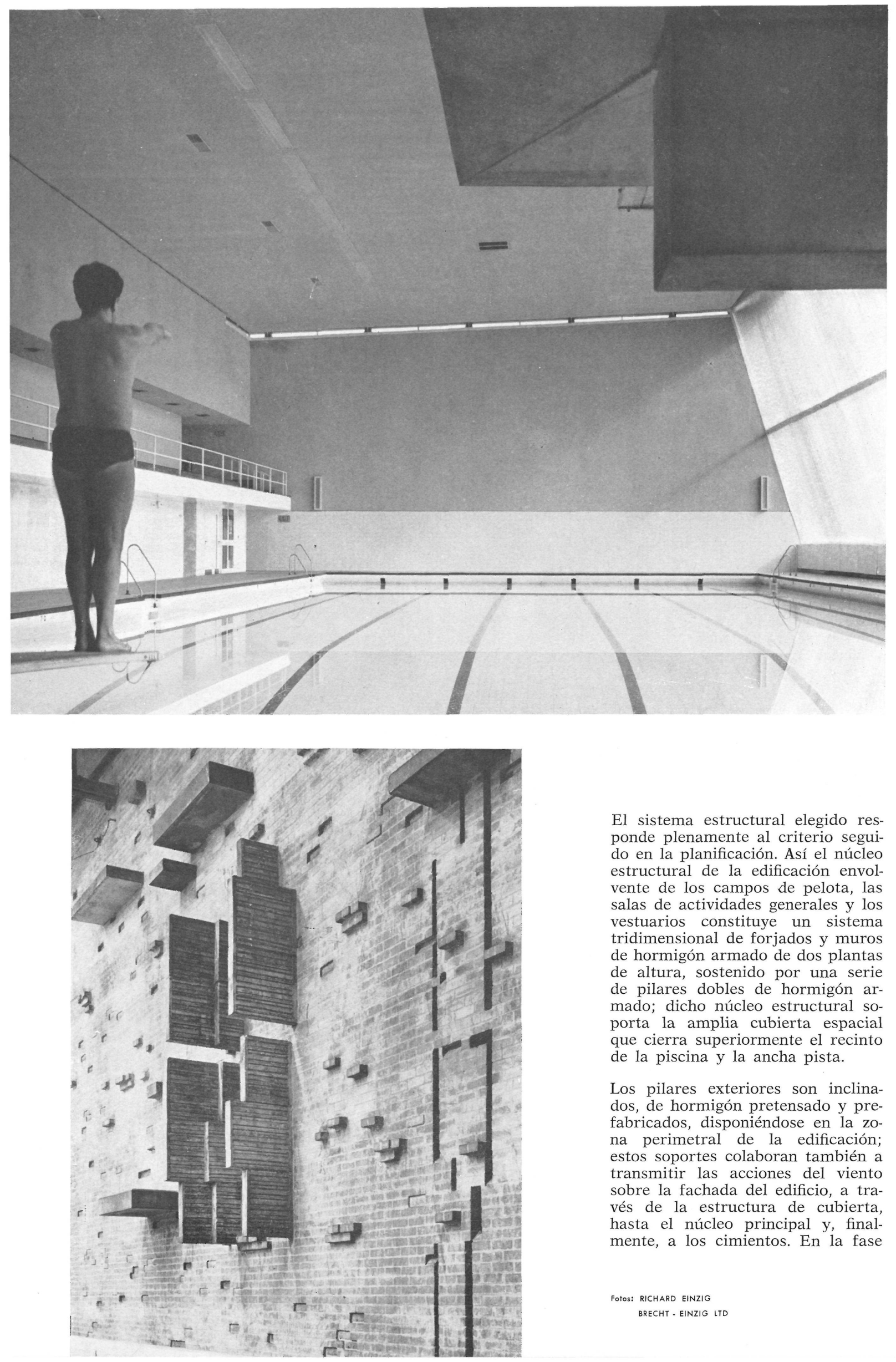

El sistema estructural elegido responde plenamente al criterio seguido en la planificación. Así el núcleo estructural de la edificación envolvente de los campos de pelota, las salas de actividades generales y los vestuarios constituye un sistema tridimensional de forjados y muros de hormigón armado de dos plantas de altura, sostenido por una serie de pilares dobles de hormigón armado; dicho núcleo estructural soporta la amplia cubierta espacial que cierra superiormente el recinto de la piscina y la ancha pista.

Los pilares exteriores son inclinados, de hormigón pretensado y prefabricados, disponiéndose en la zona perimetral de la edificación; estos soportes colaboran también a transmitir las acciones del viento sobre la fachada del edificio, a través de la estructura de cubierta, hasta el núcleo principal y, finalmente, a los cimientos. En la fase

Fotos: RICHARD EINZIG

BRECHT - EINZIG ITD 


\section{detalles}

\section{esquinas}

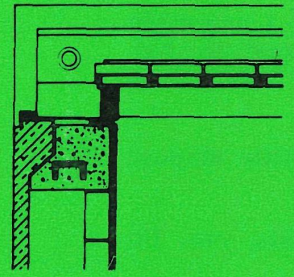

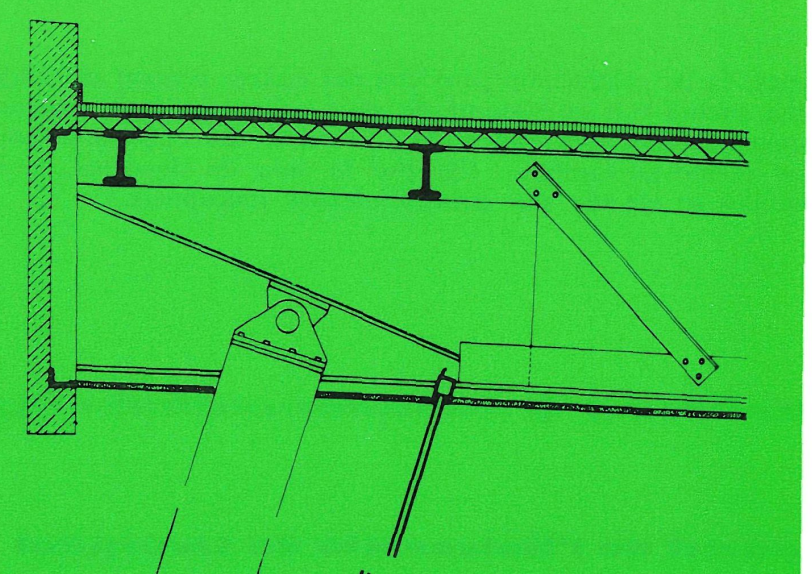

\section{fachada Iateral}

de montaje, y mediante el empleo de 2 grúas móviles, se elevó cada soporte y su correspondiente viga, enlazando posteriormente las "L" resultantes mediante conexiones metálicas.

Los muros hastiales presentan estructuras metálicas en celosía, revestidas con placas prefabricadas de hormigón visto. Las losas existentes alrededor de la pista de deportes, desde el punto de vista estructural, sirven para resistir las solicitaciones laterales resultantes de la presión del viento y las componentes horizontales de los soportes inclinados.

La estructura de la piscina fue proyectada y calculada como una estructura independiente - colgada dentro del edificio-. Ha sido construida asimismo una junta de dilatación continua alrededor de la piscina, al nivel de la primera planta, con el fin de aislar su estructura e independizarla de los movimientos generales del edificio que, de otra manera, podrían causar roturas o grietas en la misma, con las consiguientes filtraciones.

En el interior del recinto, las paredes presentan sus fábricas "vistas», o van revestidas con azulejos; los suelos han sido pavimentados a base de baldosas, o madera dura; y los cielorrasos han sido tratados acústicamente. 
Respecto a las instalaciones: la de calefacción proviene del cuarto central de calderas de la Universidad; la sala de la piscina cuenta con un sistema de agua caliente a baja presión, a base de paneles empotrados en el suelo y radiadores escondidos; el resto del edificio dispone de convectores y radiadores, controlados termostáticamente. Excepto la sala de deportes, la ventilación de las principales partes del edificio se efectúa por procedimientos mecánicos.

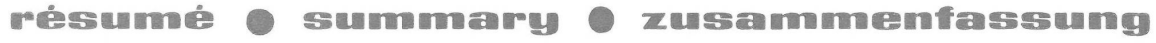

\section{Pavillon polgspontif de I"Université de Liverpool}

Denys Lasdun \& Partners, architectes

Deux zones principales se distinguent clairement: la "piscine» et la «piscine sportive». L'ensemble compte en outre quatre terrains de pelote, les salles d'activités générales pour la pratique des haltères, l'escrime, la gymnastique rythmique, le judo, la lutte, etc., les vestiaires, les bureaux, le bar, les magasins, etc.

La piscine mesure $33,53 \mathrm{~m} \times 14,63 \mathrm{~m}$; sa profondeur oscille entre $0,91 \mathrm{~m}$ et $3,80 \mathrm{~m}$. Elle dispose de plongeoirs et de tremplins de haut vol et d'une galerie pour 150 spectateurs assis.

Sur la piste polysportive, de $36,58 \mathrm{~m} \times 19,50 \mathrm{~m}$, sont pratiqués le basket-ball, le tennis, etc. Un système de filets permet de la diviser pour la pratique simultanée de plusieurs sports.

\section{Multispomts pawillion at Liverpool universitg}

Denys Lasdun \& Partners, architects

In this general project two main zones can be clearly differentiated. One is the swimming pool, and the the other the sport field. There are also four pelota courts, rooms for general activities, where weightlifting, gymnastics, rythmic dancing, judo, wrestling and other exercises can be practised, as well as offices, dressing rooms for both sexes, a bar and store rooms.

The pool is $33.53 \mathrm{~m} \times 14.63 \mathrm{~m}$ in area, and its depth varies betwen 0.91 and $3.80 \mathrm{~m}$. It has low and high diving boards, and a stand for 150 spectators, providing a good view of the swimming competitions.

The multisports field, is $36.58 \mathrm{~m} \times 19.50 \mathrm{~m}$ in area, can be used for basket ball, tennis, and other such sports. It can be divided by netting, so that several sports may be practised simultaneously.

\section{Sporthalle der Universitait Liverpool}

Denys Lasdun \& Partners, Architekten

Es gibt in ihr eine klare Trennung zwischen dem Schwimm- und dem Mehrsportbereich. Der Gesamtbau verfügt auch über vier Flächen für Ballsportarten; Mehrzweckhallen für Hanteln, Fechten, rhytmische Gymnastik, Judo, Ringen und andere der körperlichen Ertüchtigung dienenden Sportarten; Büroräume, Männer- und Frauenumkleideräume, eine Erfrischungsbar, Lager, usw.

Das Schwimmbecken misst 33,53 $\mathrm{m} \times 14,63 \mathrm{~m}$ und seine Tiefe schwankt zwischen 0,91 $\mathrm{m}$ und 3,80 $\mathrm{m}$. Es verfügt über Turm- und Brettsprunganlagen und eine Tribüine mit 150 Sitzplätzen.

Auf der Sportfläche $(36,58 \mathrm{~m} \times 19,50 \mathrm{~m})$ werden Korbball, Tennis, usw gespielt. Mit Hilfe eines Netzsystems kann man sie aufteilen, mit dem Zweck mehrere Sportarten zur gleichen Zeit auszuüiben. 